\title{
Construction of Landscape Poetry Road under the Background of Transportation and Travel Integration
}

\author{
Huang Senyan ${ }^{1}$, Qiang Rongrong ${ }^{2,3}$, Wang $\operatorname{Dan}^{2,3}$ \\ ${ }^{1}$ Hangzhou Qianhuang Expressway Co., Ltd, Zhejiang Province Hangzhou \\ 2.Research Institute of Highway Ministry of Transport; \\ ${ }^{3}$ Key Laboratory of Highway Traffic Environmental Protection Technology and Transportation Industry
}

\begin{abstract}
Under the background of the integration of transport industry and tourism industry, the highway can expand many functions such as ecology, tourism, scenic view, nostalgia and so on. By this, it replaces the traditional single road traffic function, organically combine the appreciation of natural landscape, ecological environment protection and the pursuit of human history, and closely connects the highway with the tourist area. Relying on the typical projects and the regional characteristics of rich tourism resources, this paper deeply digs the regional natural ecological elements of the "Home of Mountains and Rivers" and the regional cultural characteristics of "Millennium Poetry Road", and connects the pearls on the golden tourism line. This paper aims to study construction of the fine road of tourism traffic in mountains, rivers and poetry road under the background of the integration of traffic and tourism, and provides practical exploration and reference for the integrated development of highway and tourism.
\end{abstract}

\section{Background}

The Communist Party of China Central Committee and the State Council issued the Outline of Building a Traffic Power in September 2019 and proposed to accelerate development of new business types and new modes and deepen the integrated development of traffic and tourism. Some Opinions on Promoting the Integrated Development of Traffic and Tourism" jointly issued by six departments such as Ministry of Transport and the National Tourism Administration put forward opinions such as "improve the network system of tourism and traffic infrastructure, perfect the tourism service function of traffic service facilities, promote the innovation of tourism and traffic products and improve the quality of tourism and traffic services" and proposed the following requirements: stick to the outstanding characteristics ${ }^{\mathrm{a}}$, determine the goals, paths and measures of tourism and traffic development according to local conditions, and create a safe and green tourism and transportation boutique with local characteristics. The landscape construction of highway service areas connecting important scenic spots has been strengthened. In accordance with the requirements of beautiful landscape, strong experience and great drive, by combining with the construction of scenic spots and scenic roads, strengthen protection of ecological resources and the planning and construction of amorous towns, characteristic villages, car campsites and greenway systems along the route, and form widely influential natural scenery lines, historical humanities lines and red cultural lines. Strengthen the connection with the industry and tourism operation along the highway. Promote products and highway tourism routes, promote highquality tourism road and self-driving tour routes and guide the development of self-driving vehicles and camper tourism. Speed up the formation of a new pattern of integrated development of traffic and tourism.

This paper intends to study construction of tourism and traffic boutique roads with local characteristics under the background of transportation and tourism integration by relying on typical highway projects, explore the integration of highway and tourism in practice, and provide reference and reference.

\section{Overview of Project}

The Chun'an section of Liyang-Ningde National Expressway in Zhejiang Province (hereinafter referred to as "Qianhuang Expressway") is an important part of the national expressway network G4012 Liyang-Ningde tie line. Its starting point is near Taling at the junction of Zhejiang and Anhui and its ending point is located at the Qiandaohu branch line of Hangzhou Xinjing Expressway with a total length of $51.422 \mathrm{~km}$. This line is constructed as a standard two-way four-lane highway, the designed speed is $80 \mathrm{~km}$, and the width of roadbed is $25.5 \mathrm{~m}$. There are 5 interconnections, 43 bridges and 27 tunnels along the line.

Qiandao-Huang Expressway is located in the peripheral protection area of Qiandao Lake National Scenic Spot, Qiandao Lake National Second Drinking Water Resources Reserve and Qiandao Lake National Forest Park (5A tourist area). Therefore, construction and operation of Qianhuang Expressway have high 
requirements for ecological environment protection and water environment protection.

\section{Analysis of the Regional Characteristics of the Integration of Traffic and Tourism}

\subsection{Analysis of the Regional Characteristics of the Integration of Traffic and Tourism}

This project is located in Chun'an County, which is located in the western part of Zhejiang Province, the hilly area in the southwest of Hangzhou, between Baiji Mountains and Qianligang Mountains, and at the confluence area of Xin'an River and Qiandao Lake. It has been known as "Beautiful mountains and the hometown of mountains and rivers" since ancient times.

There are overlapping green mountains and lush forests in this territory, it is famous for its natural scenery such as forests, waterfalls and colorful rocks and has firstclass natural ecological environment, rich species of mountain and forest vegetation, and dozens of national key protected plants. The clear Xin'an River runs through the whole territory, the pearl-like Qiandao Lake is scattered and numerous streams and well-developed water systems form a characteristic scene of a thousand islands, beautiful rivers and a golden belt (a layer of golden earth belt surrounded by the island and the lake is called "Golden Belt"). In 2020, Chun'an County was named as the fourth batch of practice and innovation base of "Green Water and Green Mountain is Gold Mountain and Silver Mountain" by the Ministry of Ecology and Environment. From Qianfeng County in ancient times to Qiandao Lake today, it has formed a complex and varied landscape with rich plant resources, mountains and rocks of different shapes, beautiful and tortuous streams, ups and downs and colorful waterfalls, colorful pools and misty lakes.

From a larger scale, the constructed Qianhuang Expressway will connect famous tourist destinations such as "Famous Cities, Famous Lakes and Famous mountains" of Shanghai, Hangzhou West Lake, Qiandao Lake and Anhui Huangshan through the highway and form a "Hangzhou-Shanghai-Qiandao Lake-Huangshan" golden tourism and traffic corridor. "Qiandao Lake" is a bright pearl on this golden tourism line.

\subsection{Analysis of the Elements of Humanistic Characteristics in the Area of Traffic and Tourism Integration}

Chun'an, which has been built for more than 1800 years, has a good name of "famous nation of literature" and has profound historical and cultural heritage. Yuxiu mountains and rivers have given birth to generations of landscape poets, leaving a string of shining footprints hidden between the mountains and rivers and pieces of wellknown poems. Traffic is integrated with tourism based on the poetry, and traffic and tourism resources are consolidated based on landscape, poetry and road. By depending on Development Planning of Zhejiang Poetry
Road Cultural Belt, a high-quality tourism and traffic product with characteristics is created.

In 2019, the Zhejiang provincial government issued the "Development Plan of Zhejiang Poetry Road Cultural Belt". By comprehensively sorting of the historical, cultural and geographical resources in Zhejiang, the government proposed to take "Poetry" as the main line and "Poetry" as the finishing touch to create "Four Poetic Roads". The ancestors chose water to live and thrive business and took the water system (ancient road) as the link throughout the province. They outlined the poet's trail map, the river system traffic map, the heritage scenery map, the famous city ancient town map and the Zhejiang learning vein map of Zhejiang Poetry Road culture. They respectively drew the "four poetry roads" of the Grand Canal Poetry Road, Qiantangjiang Poetry Road, eastern Zhejiang Tang Poetry Road, and Oujiang Landscape Poetry Road.

Among them, Qiantang River Poetry Road takes Qiantang River, Fuchun River, Xin'anjiang River, Lanjiang River, Wujiang River and Qujiang River as the main lines, including Puyang River branch line and Xinanjiang Anhui Huangshan branch line and covering administrative areas such as Hangzhou, Jinhua, Quzhou and Jiaxing Haining. Qiantang Millennium Poetry Road culture takes Jin and Tang as the period of occurrence and development, Song and Yuan as the period of prosperity and prosperity, and Ming and Qing dynasties as the period of deepening integration. In the Northern Song Dynasty with the completely prosperous period of Poetry Road, the poet Liu Yong wrote, "Southeast Xingsheng, Sanwu Capital, Qiantang has been prosperous since ancient times. Smoke, willow and painting bridge, wind curtain and green curtain, and staggered 100,000 families. Cloud trees surround the embankment and sand. The raging waves roll frost and snow, and there is no limit to the graben. The city lists Zhuji, every household is full of Luoqi and Jinghao extravagance. Heavy lakes and mountains offer a good scene. There are three autumn guizi and ten miles of lotus. The picture of the different day will be a good scene and go back to Fengchi to boast." Qianhuang Expressway, which is located at the middle reaches of Xin'an River and is an important part of Qiantang River Poetry Road. By actively expanding the connotation of carriers such as landscape, humanities and industrial characteristics and integrating regional cultural characteristics such as Confucianism, Buddhism, Taoism, poetry, calligraphy, tea ceremony, opera, traditional crafts, folklore, dialects and folklore, the millennium poetry road culture is deeply planted in Zhejiang studies, thousands of villages and towns and ten cities carry the ancient rhyme of sages, and thousands of monuments are cast into a cultural treasure house. Activities such as study tourism, ancient town tourism, historic landscape eco-tourism are carried out along the theme of thousands of miles of beautiful mountains and rivers to create influential charm cultural belts, golden tourism belts, beautiful ecological belts and rich people's economic belts. 


\section{Key Points of the Construction of Landscape Poetry Road under the Integration of Traffic and Tourism}

\subsection{Line Arrangement of "Do not enter the lake near the lake and occasionally expose it"}

Qianhuang Highway is located in the scenic area of Qiandao Lake, having undulating topography, complex geological conditions, beautiful natural environment and precious land resources. We implement the design concept of "Beautiful Highway", make full use of the corridor landscape and landscape resources, and realize the beautiful vision of "Road in Green and Pedestrian in Scenery" through design and creation in the design. We should carry out adequate research on ecological protection and ecological restoration, implement the environmental protection project, and create an "Ecological Highway" during design.

In the design, by combining with the characteristics of the mountain topography, we make full use of the topographical conditions, flexibly use the technical indicators, set up the line position with the concept of "do not enter the lake near the lake and occasionally expose it", choose the design scheme based on bridges and tunnels (the proportion of bridges and tunnels is about $78 \%$ ), and reduce high filling and deep excavation and man-made damage to the natural landscape. Therefore this project is based on the concept of "do not enter the lake near the lake and occasionally expose it".

By adhering to the principle of "fill rather than dig, tunnel rather than dig, erect bridge rather than fill", we adopt design methods such as "replace roads with bridges, replace tunnels with tunnels, move and dig for filling", bridges are set up when sensitive areas of Qiandao Lake are crossed and tunnels are set up when mountains with higher heights are crossed to avoid disturbance and pollution to the water body, reduce the occupied area of the project, the amount of earthwork and high filling and deep excavation.

We optimize the line design, adhere to the principle of "no damage is the greatest protection", give priority to the avoidance scheme for environmentally sensitive points such as secondary drinking water source protection areas and national natural scenic spots along the line, achieve the goal of perfect integration and harmonious symbiosis of the highway and the natural environment, set up the line position with the concept of " do not enter the lake near the lake and occasionally expose it", and achieve "three avoidance", namely the route will avoid the core area of the "Fuchun River-Xin'anjiang" scenic spot, avoid areas prone to serious geological disasters such as landslides and debris flows, and avoid concentrated residential areas and the central area of core villages and towns. This paper aims to lay the foundation of corridor line for the ecology and environmental protection of Qianhuang highway, and realize the construction goal of integrating expressway with natural landscape environment.
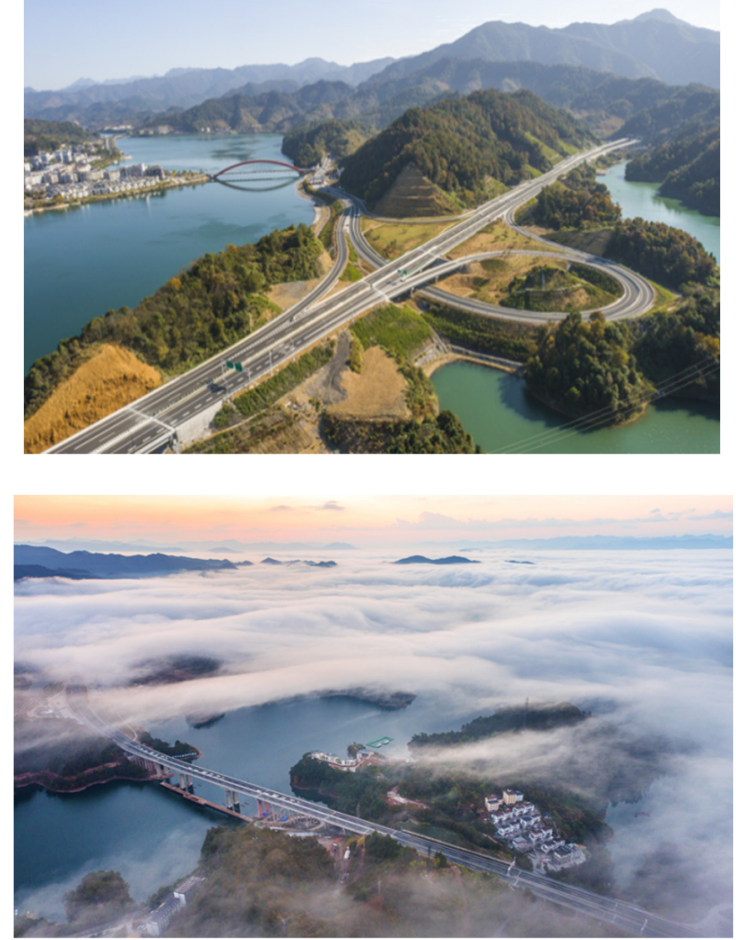

Fig. 1. Location arrangement of Qianhuang Highway (part)

\subsection{Water Environment Protection}

Zhu Xi's poem of Fangtang was written in Chun'an, which depicted "Ask the canal why to be so clear". Chun'an water environment resources are unique, 88 rivers crisscross, type I water accounts for 62 . The constructed "Zero sewage discharge Zone" does not allow a single drop of sewage to flow into Qiandao Lake, making 573 square kilometers of the lake crystal clear and visible up to 12 meters. The beautiful water of Qiandao Lake is not only the place of Chun'an modern history, but also the foundation of Chun'an future development. Therefore, the protection of water environment is one of the key points in the construction of Qianhuang Highway.

The whole line and bridge deck runoff collection and emergency disposal are used in this project, 209 runoff collection ponds are set up on the whole line, the closed collection of runoff in the road area is realized, and nearly 30 million is invested in water environmental protection measures.

The main line across the catchment area of Qiandao Lake is composed of Jinfeng Bridge and Jinfeng parking area. Jinfeng Parking Area is the only parking area on the whole line, which has a large traffic flow and is an extremely sensitive area of water environment. Construction production and domestic wastewater shall be reasonably handled during the construction period; Waste water such as oily wastewater, construction mud water and construction machinery flushing is strictly prohibited from being discharged into sensitive water bodies, domestic sewage from service facilities during the operation period is treated and reused, and outflow is prohibited.

The bridge floor runoff monitoring system is used to carry out real-time monitoring and early warning of the collected bridge deck runoff, emergency storage facilities 
are used for collection and treatment pool, a parallel mode is used for bridge floor runoff treatment facilities. The accident water and non-accident rain water runoff is disposed through the electromagnetic tee valve conversion device set up between two facilities.

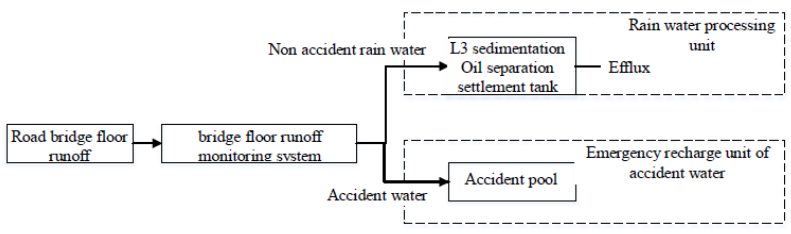

Fig. 2. Combined process flow chart of bridge floor runoff + bridge floor runoff monitoring system + collection and continuation facilities

\subsection{Landscape and Environmental Protection}

This project connects Hangzhou, Qiandao Lake and Huangshan Five-A scenic spots and there are beautiful scenery, undulating mountains and meandering rivers along the way. To drive on the road of the project, you just like being in Huangshan-Qiandao Lake Scenic area. This project implements the concept of traffic and tourism integration, takes the road as the scene, enters the country with the scene, and carries out the special design of green landscape on the basis of civil construction drawings. Thus, this project add splendor to the beautiful scenic spot. The theme of green landscape design is: between mountains and rivers.

\subsubsection{Highway greening landscape}

In the middle zone, evergreen shrubs juniper cypress and red leaf Photinia which have better anti-glare effect are used as backbone anti-glare tree species, while crape myrtle, hibiscus, Jinsen privet and safflower trees are used as middle zoning landscape plants.

At conditional protection road and broken platform position of the upper and lower slope, oleander, hibiscus, hibiscus, four seasons osmanthus, Yunnan Huangxin, Parthenocissus, Lingxiao and other ornamental and foliage plants are designed by following the principle of climbing up and down to enrich the landscape along the road. At the same time, it plays a certain role in blocking the excavation surface of the slope.

In the road sections away from the parking area, interchange and other important nodes, strengthen the middle zoning and the greening of the upper and lower slope platform, and increase the planting density and landscape tree species on the basis of the original greening such as: oleander, chicken claw maple, ginkgo, sweetscented osmanthus, red leaf heather, Yingchun, hibiscus, Phoenix tail orchid, wax plum and so on.

\subsubsection{Greening landscape in the interchange area}

The landscape greening of the interchange area is displayed through design techniques such as "topography shaping + vegetation planting" according to the characteristics of natural environment, topography and landforms, customs and customs of each interchange area in this project. Thus, the landscape of the interchange is integrated with the surrounding natural environment and cultural environment.

To create an intercommunication scene highly integrated with the surrounding environment, and effectively improve the safety factor of driving, some conditional ramp slope will be slowed down and smoothly connected with the topography within the ring, and then the road landscape will be created through the shaping of micro-topography, and smooth drainage shall be ensured.

The plants in the interchange area are selected from native plants widely distributed in the surrounding environment and suitable plants that have adapted to the local climate after long-term domestication such as phyllostachys pubescens, pinus massoniana, cunninghamia lanceolata, schima superba, liquidambar formosana, metasequoia glyptostroboides, ginkgo biloba, Ligustrum lucidum, Koeluan, melia azedarach, melia azedarach, etc.. By analyzing the visual transformation characteristics of driving direction, natural planting is carried in large area to create a comfortable visual effect, road and scenery integrated driving environment in the interworking area. Some chicken feet maple, red maple, sweet-scented osmanthus, camellia, green peach, purple leaf plum, Japanese evening cherry and other bright leaves, rich flowers and shrubs are properly embellished at the visual focus.

\subsection{Traffic and Tourism Integration Service Facilities}

\subsubsection{Setting up of tourism traffic guidance signage}

Tourism traffic guidance facilities are designed through a variety of methods in this project, including:

(1) The direction signs of the tourist area shall be set up at the intersection of the connecting roads leading to the tourist area to guide the driver to the tourist area.

(2)All the tourist attractions on a tourist highway are systematically considered, and a warning sign for the distance of the tourist area are set up in the straight-line section with a good line of sight. The number of the tourist distance notice signs for scenic spot information does not exceed three, the far end most famous scenic spot information is taken as the fixed information, the forecast is carried out from the near to the far principle.

\subsubsection{Environmentally friendly logo structure}

\section{(1)Wood-like structure}

In the road sections with good line-of-sight distance and beautiful environment, the wood-like sign structure is adopted to make traffic signs fully integrated into the environment.

(2) Truss structure

In the road section with more spare space on the side of the road, the truss traffic sign structure is selected for the guide sign of the double-column structure, which makes the supporting structure more portable and 
beautiful.

\subsubsection{Setup of observation platform in the parking area}

Build a "Fast-forward and slow tour " traffic network that meets the tourism experience, and set up parking scenic spots according to local conditions combined with landscape features and tourism resources. Two parking viewing platforms are set up in this project, which are located at the right location near the lake in Jinfeng parking area and Pingshan, respectively, and a tourism can overlook Qiandao Lake as a national 5A scenic spot on the viewing platform. Thus, tourists can fully enjoy the natural scenery and cultural landscape created by the highway. The time and space of Qiandao Lake highway landscape has the characteristics of multi-dimensionality, which connects with upper blue sky, lower lake water, has an undulating zonal space, and also contains many factors such as season, climate and other natural phenomena. The design of the viewing platform embodies the seasonality and culture of the landscape around Qiandao Lake. Thus, the landscape is coordinated in time and space.

The paving form of the scenic area is unified with the natural environment of the highway, the materials are selected by combining with the surrounding topography and environment, and different paving forms are used to divide the different functions of each area of the viewing platform. The same pavement as the highway pavement is used to highlight the integration of the viewing platform and the highway.

The design of the landscape sketch at the scenic spot not only meets the traffic function, but also pays attention to the detailed landscape design. Separating short sketches such as railings and Scenic Bridge are made of local materials, and are integrated into the nature. Functional sketches such as tables, chairs, dustbins are made of materials with proper color for harmony with surrounding landscape characteristics. Coordination with the pavement can achieve the harmony and unity of the whole viewing atmosphere; sculpture, stone stacking and other decorative sketches. The design combines with the functional orientation and cultural orientation of the poetry road, reflecting the spiritual connotation and artistic pursuit.

\subsubsection{Self-driving tour service facilities}

Qianhuangshan Highway has beautiful scenery and rich tourism resources, which provides unique conditions for self-driving tourism and sightseeing. The self-driving tourism belongs to a type of self-help tourism, which is a new form of tourism which is different from the traditional group tourism. It mainly refers to the behavior that tourists choose their own means of transport and drive or ride in non-operating vehicles to carry out tourism activities independently. It has several outstanding characteristics such as the specific nature of the means of transportation, the identity of drivers and passengers, and the autonomy of tourism arrangements. It includes self-driving sightseeing, leisure, experience, camper vacation, adventure and so on. At present, it refers to driving cars to travel in China.

Self-driving tours rely more on cross-regional and networked facilities and service layout than previous group tours. The tourist source of self-driving tour comes from key cities, urban agglomerations and economic belts, where self-driving activities are frequent and radiate to remote areas with beautiful natural scenery and prominent cultural characteristics.

However, compared with the self-driving tour in full swing, the corresponding personalized tourism service obviously lags behind. This new form of tourism is testing the related services of tourism departments and tourism, which urgently needs to be paid attention to and improved.

Take cultural landscape, architectural style, greening project and engineering appearance as carriers, dig the elements of historical culture, regional culture and natural ecological culture landscape along the highway, create characteristic highway cultural attractions and landscapes, show the culture of landscape poetry and road, meet the aesthetic demands and information demands of selfdriving travelers, and drive them to walk one stop at a time during the journey. Follow the landscapes walked by Fan Chengda, Huang Fusong, Zhu Xi and others, step on the academy they have stepped through, and recite the poems they have chanted, leaving unforgettable emotional experience and personalized poetic feelings.

\section{Conclusions}

Under the background of traffic and tourism integration, by relying on the Qianhuang Highway Project, this paper analyzes the elements of natural and cultural characteristics in the area where the highway is located, highlights the characteristics, dig the theme, and creates a safe and green landscape poetry road tourism traffic boutique with local characteristics. Through the trafficassociated theme tourism and the theme tourism to promote the traffic development, it can better promote the integrated development of the transportation industry and tourism.

\section{Contact information}

Qiang Rongrong 13693354904, Environmental Center of Research Institute of Highway Ministry of Transport

\section{Acknowledgement}

${ }^{a}$ Scientific research projects of Zhejiang Provincial Department of Communications: Technologies of green environmental protection and operation safety of lake district highway (2020-GCKY-01)

\section{References}

1. Li Junzhuo. Research and Application of Tourism Highway Landscape Planning and Design under the Background of Traffic and Tourism Integration (Sichuan Agricultural University, 2018).

2. Li Qili, Huang Bing, Wu Rui, etc. Exploration and 
Practice of Highway Traffic and Tourism Integration Development Planning based on Flow Management Concept. Traffic Engineering, 9(2020).

3. Wang Yaowen, Xie Hui. Analysis on the Main Points of the Design of Tourism Highway Safety Facilities under the Integration of Traffic and Tourism. Traffic Engineering, 8(2019).

4. Wang Wei, Xie Liangping. Application of Landscape Architecture in Highway Construction under the Background of Traffic and Tourism Integration. Green Highway, 9(2020). 